\title{
PSEUDOCOMPLEMENTS IN GROUPOIDS
}

\author{
K. NIRMALA KUMARI AMMA \\ (Received 15 June 1977; revised 7 October 1977) \\ Communicated by T. E. Hall
}

\begin{abstract}
This paper is devoted to a study of pseudocomplements in groupoids. A characterization of an intraregular groupoid is obtained in terms of prime ideals. It is proved that the set of dense elements of an intraregular groupoid $S$ with 0 is the intersection of all the maximal filters of $S$ and that the set of normal elements of an intraregular groupoid closed for pseudocomplements forms a Boolean algebra under natural operations. It is shown that the pseudocomplement of an ideal of an intraregular groupoid with 0 is the intersection of all the minimal prime ideas not containing it.
\end{abstract}

Subject classification (Amer. Math. Soc. (MOS) 1970): primary 20 L 99, 20 M 99; secondary 06 A 40.

Key words and phrases: Groupoid, intraregular, semigroup, pseudocomplement, normal element, cutcomplement, prime ideal, filter, Boolean algebra.

\section{Introduction}

A theory of pseudocomplements for semilattices has been developed by Frink (1962). The purpose of this paper is to develop a theory of pseudocomplements for groupoids. The first section contains some basic results about prime ideals and filters. The second section deals with intraregular groupoids. We obtain a characterization of an intraregular groupoid using prime ideals. The third section is devoted to a study of pseudocomplements. The concluding section is concerned with the pseudocomplement and the cutcomplement of an ideal. We prove that the set of all dense elements of an intraregular groupoid $S$ with 0 is the intersection of all the maximal filters of $S$ and that the set of normal elements of an intraregular groupoid closed for pseudocomplements forms a Boolean algebra under natural operations.

Some of the results here are analogous to results in Venkatanarasimhan (1970, 1971, 1974a, b). For standard concepts and notations used in this paper, the reader may refer to Birkhoff (1948) and Clifford and Preston (1961). We shall denote the lattice-join and lattice-meet by the symbols $\vee$ and $\wedge$ respectively. 


\section{Prime ideals and filters}

Throughout this section $S$ will denote a groupoid. By an ideal of $S$ we mean a non-empty subset $A$ of $S$ such that $S A \subseteq A$ and $A S \subseteq A$. An ideal $A$ of $S$ said to be prime if $a b \in A$ implies that $a \in A$ or $b \in A$. An ideal $A$ of $S$ is called a semiprime ideal if $a^{2} \in A$ implies that $a \in A$. A non-empty subset $A$ of $S$ is called a filter if $a, b \in A$ holds if and only if $a b \in A$. The smallest ideal (filter) containing an element $a$ of $S$ is called the principal ideal (principal filter) generated by $a$ and is denoted by $J(a)(K(a))$. A proper filter which is not contained in any other proper filter is called a maximal filter. It is easily seen that the set-union of any upper directed family of filters is a filter and that the intersection of a family of filters, whenever it is non-empty, is a filter.

TheOREM 1. A subset $A$ of $S$ is a prime ideal (minimal prime ideal) if and only if $S-A$ is a proper filter (maximal filter).

Proof. The first part of the theorem is given in Frink and Smith (1972, p. 315). We shall prove the second part.

Suppose $A$ is a minimal prime ideal of $S$. Then by the first part $S-A$ is a proper filter of $S$. Let $X$ be any proper filter of $S$ containing $S-A$. Then clearly $A \supseteq S-X$ and by the first part $S-X$ is a prime ideal. Hence $S-A=X$. It follows that $S-A$ is a maximal filter. Conversely suppose $S-A$ is a maximal filter of $S$. Then by the first part $A$ is a prime ideal. Let $B$ be any prime ideal of $S$ contained in $A$. Then $S-A \subseteq S-B$ and by the first part $S-B$ is a proper filter. Hence $S-A=S-B$ and so $A=B$. Thus $A$ is a minimal prime ideal.

THEOREM 2. If $A$ is an ideal (filter) of $S$ and $b \in S-A$, among all ideals (filters) containing $A$ and not containing $b$, there exists a maximal one.

Proof. It is easily seen that the family of all ideals containing $A$ and not containing $b$ is partially ordered by set-inclusion and that the set-union of any totally ordered subfamily is an ideal and is the least upper bound of the subfamily. Hence the first part follows by Zorn's lemma. The proof of the second part is similar.

COROLLARY 1. If $S$ has $1(0)$ any proper ideal (proper filter) of $S$ is contained in a maximal ideal (maximal filter).

As a consequence of Corollary 1 above and Theorem 1 we have the following

CoRollary 2. If $S$ has 0 , any prime ideal of $S$ contains a minimal prime ideal.

The following theorem is easily proved. 
THEOREM 3. If a prime ideal of $S$ contains the intersection of a finite number of ideals, it contains at least one of them.

For any two filters $A, B$ of $S$ we shall denote the smallest filter containing $A \cup B$ by $A \vee B$.

The following theorem is similar to Theorem 2 of Frink and Smith (1972).

THEOREM 4. If $a, b \in S, K(a) \vee K(b)=K(a b)$.

TheOREM 5. If $A, B$ are filters of $S, A \vee B=\bigcup\{K(a b): a \in A, b \in B\}$.

Proof. Let $X=\bigcup\{K(a b): a \in A, b \in B\}$. First we shall prove that $X$ is a filter. Let $x, y \in X$. Then $x \in K\left(a_{1} b_{1}\right), y \in k\left(a_{2} b_{2}\right)$ for some $a_{1}, a_{2} \in A$ and $b_{1}, b_{2} \in B$. Clearly

by Theorem 4 .

$$
\begin{aligned}
x y \in K\left(a_{1} b_{1}\right) \vee K\left(a_{2} b_{2}\right) & =K\left(a_{1}\right) \vee K\left(b_{1}\right) \vee K\left(a_{2}\right) \vee K\left(b_{2}\right) \\
& =K\left(a_{1}\right) \vee K\left(a_{2}\right) \vee K\left(b_{1}\right) \vee K\left(b_{2}\right) \\
& =K\left(a_{1} a_{2}\right) \vee K\left(b_{1} b_{2}\right) \\
& =K\left(\left(a_{1} a_{2}\right)\left(b_{1} b_{2}\right)\right)
\end{aligned}
$$

Now $a_{1} a_{2} \in A$ and $b_{1} b_{2} \in B$. It follows that $x y \in X$. Suppose $x y \in X$. Then $x y \in K\left(a^{\prime} b^{\prime}\right)$ for some $a^{\prime} \in A$ and $b^{\prime} \in B$ and so $x, y \in K\left(a^{\prime} b^{\prime}\right)$. Hence $x, y \in X$. It follows that $X$ is a filter. Let $a \in A$ and $b \in B$. Then $a \in K(a y)$ for all $y \in B$ and $b \in K(x b)$ for all $x \in A$. Hence $a, b \in X$. Thus $A \cup B \subseteq X$. If $Y$ is any filter containing $A \cup B, a \in A$ and $b \in B$, then clearly $K(a b) \subseteq Y$ and so $X \subseteq Y$. It follows that $X=A \vee B$.

Corollary. If $A$ is a filter of $S$ and $x \in S, A \vee K(x)=\bigcup_{a_{\in} A} K(a x)$.

\section{Intraregular groupoids}

A groupoid $S$ is said to be intraregular if $J(a) \cap J(b)=J(a b)$ for all $a, b \in S$. Clearly in an intraregular groupoid with $0, a b=0$ if and only if $b a=0$.

THEOREM 6. Let $A$ be an ideal of an intraregular groupoid $S$ and $b \in S-A$. Then there is a prime ideal containing $A$ and not containing $b$.

Proof. By Theorem 2, there is an ideal $M$ which is maximal among all the ideals of $S$ containing $A$ and not containing $b$. We claim that $M$ is prime. Let $x, y \in S-M$. Then $b \in(M \cup J(x)) \cap(M \cup J(y))=M \cup(J(x) \cap J(y))=M \cup J(x y)$. Consequently $x y \in S-M$, proving that $M$ is prime. 
THEOREM 7. The following statements about a groupoid $S$ are equivalent:

(1) $S$ is intraregular.

(2) Every ideal of $S$ is the intersection of all the prime ideals containing it.

(3) Every principal ideal of $S$ is the intersection of all the prime ideals containing it.

Proof. Suppose $S$ is intraregular. Let $A$ be any ideal of $S$ and $B$ the intersection of all the prime ideals containing $A$. Clearly $A \subseteq B$. Suppose $A \neq B$. Let $b \in B-A$. By Theorem 6, there is a prime ideal $M$ containing $A$ and not containing $b$. This is a contradiction to the choice of $B$. Hence $A=B$. Thus (1) implies (2).

Clearly (2) implies (3).

Suppose (3) holds and $a, b \in S$. Let $x \notin J(a b)$. Then by (3) it follows that $x \notin A$ for some prime ideal $A$ containing $a b$. Clearly $J(a) \subseteq A$ or $J(b) \subseteq A$. Hence $x \notin J(a)$ or $x \notin J(b)$ and consequently $x \notin J(a) \cap J(b)$. Thus $J(a) \cap J(b) \subseteq J(a b)$. The reverse inclusion is obvious. Hence (1).

COROLLARY 1. Every ideal of an intraregular groupoid is semiprime.

As a consequence of Theorem 7 and Corollary 2 under Theorem 2 we have the following

COROLLARY 2. In an intraregular groupoid with 0 the intersection of all the minimal prime ideals is $J(0)$.

LEMMA 1. If $S$ is an intraregular groupoid with $0, K(x)=S$ implies that $x=0$.

Proof. Suppose $x \neq 0$. Then $x \notin J(0)$. Hence by Theorem 6, there is a prime ideal $A$ containing $J(0)$ and not containing $x$. By Theorem $1, S-A$ is a proper filter. Clearly $K(x) \subseteq S-A$ and $0 \notin S-A$. It follows that $K(x) \neq S$.

LEMMA 2. If $S$ is an intraregular groupoid with 0 and $M$ is a maximal filter of $S$, $x \in S-M$ implies that ax $=0$ for some $a \in M$.

Proof. As $M$ is a maximal filter, $0 \in S=M \vee K(x)$. By the corollary under Theorem $5,0 \in K(a x)$ for some $a \in M$. Clearly $K(a x)=S$ and so by Lemma 1, $a x=0$.

\section{Pseudocomplements in groupoids}

Let $S$ be a groupoid with 0 and $a \in S$. Then by the pseudocomplement of $a$ we mean an element $a^{*}$ of $S$ such that $a a^{*}=a^{*} a=0$ and $a b=b a=0$ implies that $a^{*} b=b a^{*}=b$. Clearly the pseudocomplement of an element, whenever it exists, is unique. If every element of $S$ has a pseudocomplement, then $S$ is said to be 
closed for pseudocomplements. An element $a$ of $S$ is said to be normal (dense) if $a^{* *}=a\left(a^{*}=0\right)$. It is easily seen that an element $a$ of $S$ is normal if and only if $a=b^{*}$ for some $b \in S$.

THEOREM 8. A prime ideal $A$ of an intraregular groupoid $S$ closed for pseudocomplements is minimal prime if and only if

(*) A contains precisely one of $x, x^{*}$ for every $x \in S$.

Proof. Suppose $A$ is minimal prime. Since $x x^{*}=0 \in A, x \in A$ or $x^{*} \in A$. Suppose $x \in A$. Then $(S-A) \vee K(x)=S$ since $S-A$ is a maximal filter by Theorem 1 . By Lemma 2, $a x=0$ for some $a \in S-A$. Now $a x^{*}=a$. It follows that $x^{*} \in S-A$.

Conversely suppose (*) holds and $x \in A$. Then $x^{*} \in S-A$. Hence

$$
0=x x^{*} \in(S-A) \vee K(x) .
$$

Thus $S-A$ is a maximal filter and consequently $A$ is a minimal prime ideal.

COROLLARY. A filter of an intraregular groupoid $S$ closed for pseudocomplements is maximal if and only if it contains precisely one of $x, x^{*}$ for every $x \in S$.

LEMMA 3. If $S$ is an intraregular groupoid closed for pseudocomplements, $a, b \in S, J(a)=J(b)$ implies that $a^{*}=b^{*}$.

Proof. Let $J(a)=J(b)$. Then $J\left(a b^{*}\right)=J(a) \cap J\left(b^{*}\right)=J(b) \cap J\left(b^{*}\right)=J\left(b b^{*}\right)=J(0)$. Hence $a b^{*}=0$ and so $a^{*} b^{*}=b^{*}$. Similarly $a^{*} b^{*}=a^{*}$. Thus $a^{*}=b^{*}$.

THEOREM 9. Let $S$ be a groupoid closed for pseudocomplements. Then

(i) $a^{*} a^{*}=a^{*}$, for all $a \in S$;

(ii) $a a^{* *}=a^{* *} a=a$ for all $a \in S$;

(iii) $S$ has the identity element 1 and $0^{*}=1$;

(iv) $1^{*}=0$.

If $S$ is intraregular, then

(v) $a^{* * *}=a^{*}$ for all $a \in S$;

(vi) $a b=a$ implies that $a^{*} b^{*}=b^{*} a^{*}=b^{*}$ for $a, b \in S$;

(vii) $(a b)^{*}=\left(a^{* *} b^{* *}\right) *$ for all $a, b \in S$;

(viii) $(a a)^{*}=a^{*}$ for all $a \in S$;

(ix) $(a b)^{*}=(b a)^{*}$ for all $a, b \in S$;

(x) $(a(b c))^{*}=((a b) c)^{*}$ for all $a, b, c \in S$.

If further $S$ is an intraregular semigroup,

(xi) $(a b)^{* *}=a^{* *} b^{* *}$ for all $a, b \in S$;

(xii) $\left(a^{*} b^{*}\right)^{* *}=a^{*} b^{*}$ for all $a, b \in S$;

(xiii) $a^{*} b^{*}=b^{*} a^{*}$ for all $a, b \in S$. 
Proof (i) $a b=b a=0$ implies that $a^{*} b=b$. Taking $b=a^{*}$ we get (i).

(ii) Since $a^{*} a=a a^{*}=0$, by the definition of $a^{* *}$ it follows that

$$
a a^{* *}=a^{* *} a=a .
$$

(iii) $0 a=a 0=0$ for all $a \in S$. Hence $0^{*} a=a 0^{*}=a$. Hence (iii).

(iv) is obvious.

(v) Let $A$ be any prime ideal of $S$. Then $a \in S-A$ implies that $a^{* *} \in S-A$ which implies that $a^{* * *} \in A$. Hence all the prime ideals of $S$ contain $a a^{* * *}$ and so $a a^{* * *}=0$. Consequently $a^{*} a^{* * *}=a^{* * *}$. Now the result follows by (ii).

(vi) Suppose $a b=a$. Then

$$
\begin{aligned}
J\left(a b^{*}\right) & =J\left(b^{*} a\right)=J(a) \cap J\left(b^{*}\right)=J(a b) \cap J\left(b^{*}\right) \\
& =J(a) \cap J(b) \cap J\left(b^{*}\right)=J(0) .
\end{aligned}
$$

Hence $a b^{*}=b^{*} a=0$ so that $a^{*} b^{*}=b^{*} a^{*}=b^{*}$.

(vii) Let $A$ be any minimal prime ideal of $S$. Then $\left(a^{* *} b^{* *}\right)^{*} \in A$ if and only if $a^{* *} b^{* *} \in S-A$ if and only if $a^{* *}, b^{* *} \in S-A$ if and only if $a^{*}, b^{*} \in A$ if and only if $a, b \in S-A$ (by Theorem 8 ) if and only if $a b \in S-A$ if and only if $(a b)^{*} \in A$. Hence $\left(a^{* *} b^{* *}\right)(a b)^{*},(a b)\left(a^{* *} b^{* *}\right)^{*} \in A$. By Corollary 2 under Theorem 7 it follows that $\left(a^{* *} b^{* *}\right)(a b)^{*}=(a b)\left(a^{* *} b^{* *}\right)^{*}=0$. Consequently

$$
(a b)^{*}=\left(a^{* *} b^{* *}\right)^{*}(a b)^{*}=\left(a^{* *} b^{* *}\right)^{*} \text {. }
$$

Since $J(a a)=J(a), J(a b)=J(b a)$ and $J(a(b c))=J((a b) c)$, (viii), (ix) and (x) follow by Lemma 3.

(xi) As in (vii), $(a b)^{*}\left(a^{* *} b^{* *}\right)=0$. Suppose $(a b)^{*} c=0$ for some $c \in S$ and let $A$ be any minimal prime ideal of $S$. Then $c \in S-A$ implies that $(a b)^{*} \in A$ and hence $a b \in S-A$ (by Theorem 8). This further implies that $a, b \in S-A$ and hence $a^{*}, b^{*} \in A$. Consequently $a^{*} c, b^{*} c \in A$. By Corollary 2 under Theorem 7 it follows that $a^{*} c=b^{*} c=0$. Hence $a^{* *} c=b^{* *} c=c$, so that

This proves (xi).

$$
\left(a^{* *} b^{* *}\right) c=a^{* *}\left(b^{* *} c\right)=a^{* *} c=c .
$$

(xii) is obtained by replacing $a$ by $a^{*}, b$ by $b^{*}$ in (xi) and using (v).

(xii) is an immediate consequence of (ix) and (xii).

The following theorem is easily proved.

THEOREM 10. In (v) of Theorem 9, intraregularity of $S$ can be replaced by associativity, in (vi) and (vii) intraregularity can be replaced by associativity and commutativity and in (x) and (xi) intraregularity can be replaced by commutativity. Further if $S$ is a commutative semigroup closed for pseudocomplements the set $N$ of normal elements of $S$ forms a Boalean algebra which is a sub-pseudocomplemented subsemigroup of $S$. 
THEOREM 11. The set $D$ of all dense elements of an intraregular groupoid $S$ with 0 forms a filter of $S$ and is the intersection of all the maximal filters of $S$.

Proof. Let $A$ be the intersection of all the maximal filters of $S$. If $x \in S-A$, then $x \in S-M$ for some maximal filter $M$ of $S$. By Lemma $2, a x=0$ for some $a \in M$. Consequently $x \in S-D$. On the other hand, if $y \in S-D$, then $y z=0$ for some $z \neq 0$. By Lemma 1 and Corollary 1 under Theorem 2 it follows that $z \in M_{1}$ for some maximal filter $M_{1}$. Clearly $y \in S-M_{1}$ and so $y \in S-A$. Hence the theorem.

THEOREM 12. The set $N$ of normal elements of an intraregular groupoid $S$ closed for pseudocomplements forms a Boolean algebra under the unary operation* and the binary operation $\wedge$ defined by $a \wedge b=(a b)^{* *}$.

Proof. Let $a, b, c \in N$. Then by (viii) and (ix) of Theorem 9 it follows that $a \wedge a=a$ and $a \wedge b=b \wedge a$. Also

$$
\begin{aligned}
(a \wedge(b \wedge c)) & =\left(a(b c)^{* *}\right)^{* *}=\left(a^{* *}(b c)^{* *}\right)^{* *}=(a(b c))^{* *}=((a b) c)^{* *} \\
& =\left((a b)^{* *} c^{* *}\right)^{* *}=\left((a b)^{* *} c\right)^{* *}=((a \wedge b) \wedge c)
\end{aligned}
$$

using (vii) and (x) of Theorem 9. Clearly if $a \wedge b^{*}=0$ then $a b^{*}=0$ and hence $a b^{* *}=a$. It follows that $a b=a$ and hence $a \wedge b=a$. Suppose $a \wedge b=a$ and let $A$ be any minimal prime ideal of $S$. Then if $b^{*} \notin A$ then $b \in A$ and hence $a b \in A$. Therefore $a \wedge b \in A$ which implies that $a \in A$. Hence $a b^{*} \in A$ and so $a \wedge b^{*} \in A$. Thus all the minimal prime ideals of $S$ contain $a \wedge b^{*}$ and so by Corollary 2 under Theorem $7 a \wedge b^{*}=0$. Now the theorem follows by a well-known characterization of Boolean algebra due to Frink (1941).

Whether or not $N$ is a subgroupoid of $S$ in Theorem 12 is an open problem. However, we have the following

THEOREM 13. Let $S$ be an intraregular groupoid closed for pseudocomplements. Then the following statements are equivalent:

(1) $(a b)^{* *}=a^{* *} b^{* *}$ for all $a, b \in S$;

(2) $a^{*}\left(b^{*} c^{*}\right)=\left(a^{*} b^{*}\right) c^{*}$ for all $a, b, c \in S$;

(3) $a^{*} b^{*}=\left(a^{*} b^{*}\right)^{* *}$ for all $a, b \in S$.

Proof. Suppose (1) holds. By (x) of Theorem 9 it follows that

that is,

$$
\left(a^{*}\left(b^{*} c^{*}\right)\right)^{* *}=\left(\left(a^{*} b^{*}\right) c^{*}\right)^{* *}
$$

$$
a^{* * *}\left(b^{*} c^{*}\right)^{* *}=\left(a^{*} b^{*}\right)^{* *} c^{* * *}
$$


by (1). Using (1) again we have

$$
a^{* * *}\left(b^{* * *} c^{* * *}\right)=\left(a^{* * *} b^{* * *}\right) c^{* * *} .
$$

Now (2) follows by (v) of Theorem 9.

Suppose (2) holds. Then $a^{*}\left(a^{*} b^{*}\right)=\left(a^{*} a^{*}\right) b^{*}=a^{*} b^{*}$ by (i) of Theorem 9 . Hence by (v) and (vi) of Theorem $9 a^{*}\left(a^{*} b^{*}\right)^{* *}=\left(a^{*} b^{*}\right)^{* *}$. Similarly $b^{*}\left(b^{*} a^{*}\right)^{* *}=\left(b^{*} a^{*}\right)^{* *}$; that is, $b^{*}\left(a^{*} b^{*}\right)^{* *}=\left(a^{*} b^{*}\right)^{* *}$ by (ix) of Theorem 9 . Hence by (2) $\left(a^{*} b^{*}\right)\left(a^{*} b^{*}\right)^{* *}=a^{*}\left(b^{*}\left(a^{*} b^{*}\right)^{* *}\right)=a^{*}\left(a^{*} b^{*}\right)^{* *}=\left(a^{*} b^{*}\right)^{* *}$. Also by (ii) of Theorem $9\left(a^{*} b^{*}\right)\left(a^{*} b^{*}\right)^{* *}=a^{*} b^{*}$. Hence (3).

Replacing $a$ by $a^{*}, b$ by $b^{*}$ in (3) and using (vii) of Theorem 9 we get (1). Thus (3) implies (1).

\section{Pseudocomplement of an ideal}

If $S$ is a groupoid with 0 the set of all ideals of $S$ forms a pseudocomplemented completely distributive complete lattice under set-inclusion as ordering; the join and meet coincide with set-union and set-intersection. A normal (dense) element of this lattice is called a normal ideal (dense ideal). The cutcomplement $A_{c}$ of an ideal (filter) $A$ of a groupoid $S$ is defined by $A_{c}=\bigcap_{a \in A} K(a)\left(\bigcap_{a \in A} J(a)\right)$. An ideal of $S$ which is an intersection of principal ideals is called a comprincipal ideal.

THEOREM 14. A prime ideal of a groupoid $S$ with 0 is either dense or normal.

Proof. Let $A$ be a prime ideal of $S$. Then $A \supseteq A^{*}$ or $A \supseteq A^{* *}$ by Theorem 3 . It follows that $A^{*}=J(0)$ or $A=A^{* *}$.

The following theorem is easily proved.

THEOREM 15. If $A$ is an ideal of a groupoid $S$ with $0, A^{*}=\{x \in S: A \cap J(x)=J(0)\}$. If $S$ is intraregular, $A^{*}=\{x \in S: a x=0$ for all $a \in A\}$ and $J(a)^{*}=\{x \in S: a x=0\}$.

THEOREM 16. In an intraregular groupoid closed for pseudocomplements, $J(x)^{*}=J\left(x^{*}\right)$ for every $x$.

Proof. Now $J(x) \cap J\left(x^{*}\right)=J\left(x x^{*}\right)=J(0)$ and so $J\left(x^{*}\right) \subseteq J(x)^{*}$. Also $y \in J(x)^{*}$ implies that $x y=0$ (by Theorem 15) which implies that $x^{*} y=y$ and hence $y \in J\left(x^{*}\right)$. Thus $J(x)^{*} \subseteq J\left(x^{*}\right)$. It follows that $J(x)^{*}=J\left(x^{*}\right)$.

THEOREM 17. A prime ideal $A$ of an intraregular groupoid $S$ with 0 is minimal prime if and only if

(*) A contains precisely one of $J(x), J(x)^{*}$ for every $x \in S$. 
Proof. Suppose $A$ is minimal prime. By Theorem $3, A$ contains at least one of $J(x), J(x)^{*}$. Suppose $A \supseteq J(x)$. Then $(S-A) \vee K(x)=S$ since $S-A$ is a maximal filter by Theorem 1. By Lemma $2, a x=0$ for some $a \in S-A$. Hence by Theorem 15, $a \in J(x)^{*}$ and so $J(x)^{*} \nsubseteq A$.

Conversely suppose $\left(^{*}\right)$ holds and $x \in A$. Then $J(x)^{*} \ddagger A$. Let $y \in J(x)^{*}-A$. Then $0=x y \in(S-A) \vee K(x)$. It follows that $S-A$ is a maximal filter and consequently $A$ is a minimal prime ideal.

COROLlary. If $M$ is a minimal prime ideal of an intraregular groupoid, $x \in M$ implies that $J(x)^{* *} \subseteq M$.

As an immediate consequence of (vi) of Theorem 9 we have the following

THEOREM 18. If $A, B$ are ideals of a groupoid $S$ with $0 ; A \subseteq B$ implies that $A^{*} \supseteq B^{*}$.

THEOREM 19. If $A$ is an ideal of an intraregular groupoid with $0, A^{*}=\bigcap_{a \in A} J(a)^{*}$.

ProOf. Let $x \in \bigcap_{a \in A} J(a)^{*}$. Then $x \in J(a)^{*}$ for every $a \in A$. Hence by Theorem 15 $x \in A^{*}$. Thus $\bigcap_{a \in A} J(a)^{*} \subseteq A^{*}$. The reverse inclusion follows by Theorem 18 .

COROLLARY. In an intraregular groupoid closed for pseudocomplements, every normal ideal is comprincipal.

THEOREM 20. The pseudocomplement of an ideal $A$ of an intraregular groupoid $S$ with 0 is the intersection of all the minimal prime ideals of $S$ not containing $A$.

Proof. Let $B$ be the intersection of all the minimal prime ideals not containing $A$. Clearly $A^{*} \subseteq B$. Suppose $A^{*} \neq B$. Let $b \in B-A^{*}$. Then by Theorem $15, a b \neq 0$ for some $a \in A$. By Lemma $1, K(a b)$ is a proper filter of $S$. Hence by Corollary 1 under Theorem $2, K(a b) \subseteq M$ for some maximal filter $M$ of $S$. Now $a b \in M$ and so $a, b \in M$. Hence $A, B \nsubseteq S-M$. Also $S-M$ is a minimal prime ideal by Theorem 1 . Thus we get a contradiction to the choice of $B$. Hence the theorem.

COROLLARY. The pseudocomplement of an ideal $A$ of an intraregular groupoid $S$ with 0 is the intersection of all the prime ideals not containing $A$.

THEOREM 21. Let $S$ be an intraregular groupoid with 0 . Then any normal ideal of $S$ is the intersection of all the minimal prime ideals containing it and a principal ideal of $S$ is normal if it is the intersection of all the minimal prime ideals containing it.

ProOF. The first part is an immediate corollary of Theorem 20. Let $J(a)$ be a principal ideal of $S$ such that $J(a)=\bigcap_{i \in I} M_{i}$ where $\left\{M_{i}: i \in I\right\}$ is the family of all 
the minimal prime ideals containing $J(a)$. Then $a \in M_{i}$ for all $i \in I$ and so by the corollary under Theorem $17, J(a)^{* *} \subseteq M_{i}$ for all $i \in I$. Hence

$$
J(a)^{* *} \subseteq \bigcap_{i \in I} M_{i}=J(a) .
$$

It follows that $J(a)$ is normal.

THEOREM 22. The cutcomplement of an ideal (filter) $A$ of a groupoid $S$ is the intersection of all the filters (ideals) intersecting $A$.

Proof. Let $A$ be an ideal of $S$ and $A_{1}$ the intersection of all the filters of $S$ intersecting $A$. Clearly $A_{1} \subseteq A_{c}$. Also if $B$ is any filter intersecting $A$ and $x \in A \cap B$, then $x \in A, B$ and so $A_{c} \subseteq K(x) \subseteq B$. Hence the first part. The second part is proved on similar lines.

As an immediate consequence of Theorems 7 and 22 we have the following

THEOKEM 23. If $A$ is a filter of an intraregular groupoid $S$, then $A_{c}$ is the intersection of all the prime ideals intersecting $A$.

THEOREM 24. If $A$ is an ideal of an intraregular groupoid $S$, then $A_{c c}$ is the intersection of all the principal ideals containing $A$.

Proof. It is enough to show that $x \in A_{c}$ if and only if $J(x) \supseteq A$. Suppose $x \notin A_{c}$. Then $x \notin K(a)$ for sume $a \in A$. Now $S-K(a)$ is a prime ideal by Theorem 1 and $J(x) \subseteq S-K(a)$. Clearly $J(x) \cap K(a)=\varphi$ and so $a \notin J(x)$. Thus $J(x) \neq A$. On the other hand, if $J(x) \notin A$ and $a \in A-J(x)$, then $J(x) \cap K(a)=\varphi$. Hence $x K(a)$ and so $x \notin A_{c}$.

THEOREM 25. If $A$ is an ideal of an intraregular groupoid closed for pseudocomplements, $A_{c c}^{*}=A^{*}$.

PROOF. By Theorem 24 and the corollary under Theorem 19 it follows that $A \subseteq A_{c c} \subseteq A^{* *}$. Hence by Theorem $18, A^{*} \subseteq A_{c c}^{*} \subseteq A^{*}$. It follows that $A_{c c}^{*}=A^{*}$.

\section{Acknowledgement}

The author is indebted to Dr. P. V. Venkatanarasimhan for his guidance and help in preparing this paper. She is thankful to the referee for some suggestions.

\section{References}

G. Birkhoff (1948), Lattice Theory, Colloq. Publ. 25 (Amer. Math. Soc., Providence, R.I.).

A. H. Clifford and G. B. Preston (1961), "The algebraic theory of semigroups, I", Math. Surveys 7 (Amer. Math. Soc.). 
O. Frink (1941), "Representation of Boolean algebras", Bull. Amer. Math. Soc. 47, 755-756.

O. Frink (1962), "Pseudocomplements in semilattices", Duke Math. J. 29, 505-514.

O. Frink and R. S. Smith (1972), "On the distributivity of the lattice of filters of a groupoid", Pacific J. Math. 42, 313-322.

P. V. Venkatanarasimhan (1970), "Semi-ideals in posets", Math. Ann. 185, 338-348.

P. V. Venkatanarasimhan (1971), "Pseudocomplements in posets", Proc. Amer. Math. Soc. 28, 9-17.

P. V. Venkatanarasimhan (1974a), "Semi-ideals in semilattices", Colloq. Math. 30, 203-212.

P. V. Venkatanarasimhan (1974b), "Ideals in pscidocomplemented lattices and semilattices", Acta Sci. Math. (Szeged) 36, 221-226.

Department of Mathematics

University of Kerala

Kariavattom

Trivandrum 